\title{
Transport and reaction characteristics in flue gas desulfurization
}

\author{
Y. Yan ${ }^{a}$, X.F. Peng ${ }^{a, *}$, D.J. Lee ${ }^{b}$ \\ a Department of Thermal Engineering, Tsinghua University, Beijing 100084, People Republic of China \\ ${ }^{\mathrm{b}}$ Department of Chemical Engineering, Taiwan University, Taipei 106, Taiwan \\ Received 27 May 2002; accepted 7 January 2003
}

\begin{abstract}
The transport and reaction characteristics of the product layer formation process in flue gas desulfurization were investigated theoretically and experimentally. The transport and reaction processes were described by the Thiele number incorporating the influence of the chemical reaction resistance and the diffusion resistance. The results show that the conversion rate at the end of the process decreased with increasing Thiele number and increased with increasing temperature and porosity. The conversion rate also depends on the pore geometry and pore size distribution.
\end{abstract}

(C) 2003 Éditions scientifiques et médicales Elsevier SAS. All rights reserved.

Keywords: Gas-solid reactions; Desulfurization; Mass transfer; Thiele number; Porosity

\section{Introduction}

The utilization of coal brings with it serious pollution problems, such as $\mathrm{SO}_{2}$ emission which must be reduced [1].

Several $\mathrm{SO}_{2}$ emission control technologies have been developed and applied in coal-fired power plants. Both dry flue gas desulfurization and furnace dry injection, which use solid alkaline powder as an absorbent and react with $\mathrm{SO}_{2}$, are important $\mathrm{SO}_{2}$ emission control technologies with relatively low initial investment, less corrosion and lower energy requirements, especially for older plants which need to be retrofitted at low capital cost. The low utilization rate of the sorbent is the main disadvantage of those processes which restricts their practical applications [1]. Several technologies were introduced to improve the sorbent utility such as steam reactivation [2,3] and additives [4]. However, these improvements consume additional energy or incur extra cost. Engineers are still seeking more efficient technologies which use less energy or additives.

Both dry flue gas desulfurization and furnace dry injection involve typical gas solid reactions. The product covers the sorbent surface and forms a product layer during the process. The $\mathrm{SO}_{2}$ then has to diffuse through the product layer to react with the sorbent [5]. However, some research

\footnotetext{
* Corresponding author.

E-mail address: pxf-dte@mail.tsinghua.edu.cn (X.F. Peng).
}

has suggested that the $\mathrm{Ca}^{2+}$ may diffuse out through the product layer to react with the $\mathrm{SO}_{2}$ [6]. In any case, the formation of the product layer restricts the reaction rate. In most practical applications, the $\mathrm{SO}_{2}$ absorption is most important as the product layer forms. This paper analyzes the transport and reaction characteristics during the product layer formation process.

\section{Theoretical analysis}

\subsection{Reactions mechanism in porous solids}

The gaseous reactant diffuses into the pores inside the solid reactant to react with the solid reactant. If the chemical reaction rate is far less than the diffusion rate, the reaction will occur in an environment with essentially uniform gas reactant concentration which can be analyzed with the normal volumetric reaction model. However, when the chemical reaction is much faster than the diffusion, a reaction interface forms inside the particle which separates the particle into two regions, the product layer and the reaction zone. The reaction interface moves toward the center of the particle during the reaction process until the solid reactant is completely transformed into the product. This case can be analyzed with the shrinking unreacted core model. However, most gas-solid reactions fall between these two extremes in a zone with some thickness where the solid 


\begin{abstract}
Nomenclature
$a, b, c, d$ stoichiometric coefficient

$C_{A} \quad$ gas reactant concentration $\ldots \ldots \ldots . . . \mathrm{mol} \cdot \mathrm{m}^{-3}$

$C_{A 0} \quad$ gas reactant concentration in the bulk $\mathrm{mol} \cdot \mathrm{m}^{-3}$

$C_{A r_{0}} \quad$ gas reactant concentration at the particle

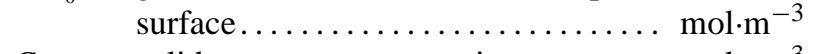

$\begin{array}{lll}C_{B} & \text { solid reactant concentration } \ldots \ldots \ldots & \mathrm{mol} \cdot \mathrm{m}^{-3}\end{array}$

$\begin{array}{lll}C_{B 0} & \text { initial solid reactant concentration } \ldots \mathrm{mol} \cdot \mathrm{m}^{-3}\end{array}$

$c_{1}, c_{2}$ integration constant in Eq. (13)

$\mathrm{De} \quad$ effective diffusion coefficient of $\mathrm{SO}_{2} \ldots \mathrm{m}^{2} \cdot \mathrm{s}^{-1}$

$k_{v} \quad$ volumful reaction rate constant. $\mathrm{m}^{3} \cdot \mathrm{s}^{-1} \cdot \mathrm{mol}^{-1}$

$r_{0} \quad$ initial particle radius $\ldots \ldots \ldots \ldots \ldots \ldots m$

$r_{s} \quad$ reactant rate constant for solid

reactant ............... $\mathrm{m}^{3} \cdot \mathrm{s}^{-1} \cdot \mathrm{mol}^{-1}$

Sh Sherwood number

$T \quad$ reaction temperature.
\end{abstract}

reactant and the solid product co-exist. Ishada [7] and Wen [8] proposed a dual-zone reaction model to described the process. In their model, the reaction process was divided into two stages, the surface reactions with product layer formation stage and the product layer and reaction region co-existing stage. In the first stage, gas reactant diffuses through the pores in the sorbent particles and reacts with the solid sorbent to form a surface product layer covering the whole surface. In the second stage, the particles are divided into two zones, the product layer and the reaction zone. The gas reactant must diffuse through the product layer and then into the reaction zone. The product layer thickness gradually increases until the entire solid reactant particle is transformed into product $[9,10]$. In practical applications, the desulfurization reaction usually lasts a very short time so more attention should focused on the first stage [1].

\subsection{Transport and reaction kinetics}

Consider a chemical reaction between a gas reactant (A) and a solid reactant $(\mathrm{B})$

$a A(g)+b B(s)=c C(g)+d D(s)$

The solid reactant concentration is defined as the mass per volume of solid reactant. The analysis is based on the following assumptions:

(1) global solid reactant;

(2) irreversible reaction;

(3) first-order kinetics reaction;

(4) pseudo-steady state diffusion; and

(5) an isothermal system.

The proposed physical model is shown in Fig. 1.

The gas reactant diffuses into the inner holes of the particle to react with the solid reactant during the formation $t \quad$ time $\ldots \ldots \ldots \ldots \ldots \ldots \ldots \ldots \ldots \ldots \ldots \ldots \ldots \ldots$

$t_{c} \quad$ the product layer formation length $\ldots \ldots \ldots \mathrm{s}$

$x_{B} \quad$ solid reactant conversion rate

$x_{c} \quad$ final product layer formation conversion rate

\section{Greek letters}

$\alpha_{D} \quad \mathrm{SO}_{2}$ convection mass transfer coefficient in the boundary layer $\ldots \ldots \ldots \ldots \ldots \ldots \ldots . m . \mathrm{s}^{-1}$

$\xi \quad$ dimensionless radius

$\theta \quad$ dimensionless time

$\theta_{c} \quad$ dimensionless time for the product layer formation process

$\psi \quad$ dimensionless gas reactant concentration

$\varphi \quad$ Thiele number

$\chi_{B} \quad$ dimensionless solid reactant concentration

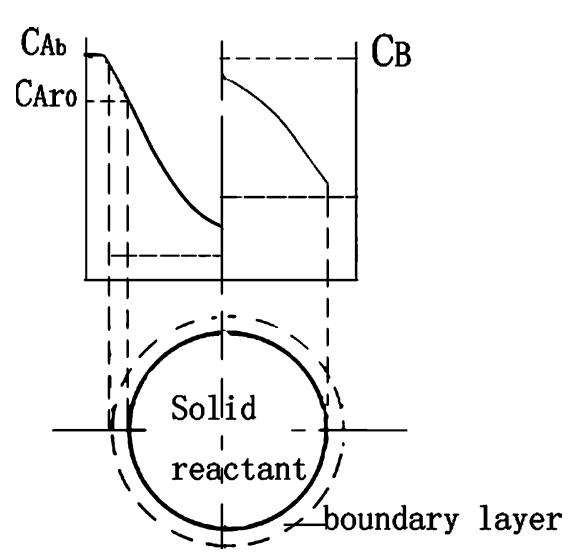

Fig. 1. Schematic diagram of concentration distributions.

of the product layer. The gas reactant concentration in the reaction zone can be described by

$\frac{D_{e}}{r^{2}} \frac{\mathrm{d}}{\mathrm{d} r}\left(r^{2} \frac{\mathrm{d} C_{A}}{\mathrm{~d} r}\right)-r_{s}=0$

where $r_{s}$ is the local chemical reaction rate defined as

$r_{s}=-\frac{\partial C_{B}}{\partial t}=k_{v} C_{B 0} C_{A}$

The boundary conditions are

$\left.D_{e} \frac{\mathrm{d} C_{A}}{\mathrm{~d} r}\right|_{r=r_{0}}=\alpha_{D}\left(C_{A 0}-C_{A r_{0}}\right)$

$\left.\frac{\mathrm{d} C_{A}}{\mathrm{~d} r}\right|_{r=0}=0$

$t=0, \quad C_{B}=C_{B 0}$

With the dimensionless parameters, $\xi=r / r_{0}$ and $\psi=$ $C_{A} / C_{A 0}$, Eqs. (2), (4) and (5) can be written as:

$\frac{1}{\xi^{2}} \frac{\mathrm{d}}{\mathrm{d} \xi}\left(\xi^{2} \frac{\mathrm{d} \psi}{\mathrm{d} \xi}\right)-\varphi^{2} \psi=0$
$\left.\frac{\mathrm{d} \psi}{\mathrm{d} \xi}\right|_{\xi=1}=\operatorname{Sh}\left(1-\psi_{\xi=1}\right)$ 
$\left.\frac{\mathrm{d} \psi}{\mathrm{d} \xi}\right|_{\xi=0}=0$

where the Thiele number, $\varphi$, and the Sherwood number, $S h$, are defined by

$\varphi=r_{0}\left(\frac{a k_{v} C_{B 0}}{b D_{e}}\right)^{1 / 2}$

$S h=\frac{\alpha_{D} r_{0}}{D_{e}}$

By introducing $z=\psi \xi$, Eq. (7) can be written as

$\frac{\mathrm{d}^{2} z}{\mathrm{~d} \xi^{2}}=\varphi^{2} z$

whose solution is $z=c_{1} \exp (\varphi \xi)+c_{2} \exp (-\varphi \xi)$ or

$\psi=c_{1} \frac{\exp (\varphi \xi)}{\xi}+c_{2} \frac{\exp (-\varphi \xi)}{\xi}$

For the boundary conditions given in Eqs. (8) and (9),

$\psi=\frac{1}{\theta_{c}} \frac{\operatorname{sh}(\varphi \xi)}{\xi \operatorname{sh}(\varphi)}$

where $\theta_{c}=1+\frac{\varphi \operatorname{cth}(\varphi)-1}{S h}$ is the dimensionless reaction time of the product layer formation process. The solid reactant concentration is given by

$$
\begin{aligned}
C_{B} & =C_{B 0}-\frac{b}{a} \int_{0}^{t} r_{s} \mathrm{~d} t \\
& =C_{B 0}-k_{v} C_{B 0} C_{A b} \frac{b}{a} \frac{1}{\theta_{c}} \frac{\operatorname{sh}(\varphi \xi)}{\xi \operatorname{sh}(\varphi)} t
\end{aligned}
$$

or

$$
\begin{aligned}
\chi_{B} & =\frac{C_{B}}{C_{B 0}}=1-\frac{b}{a} \frac{\operatorname{sh}(\varphi \xi)}{\xi \operatorname{sh}(\varphi)} \frac{k_{v} C_{A b} t}{\theta_{c}} \\
& =1-\frac{\operatorname{sh}(\varphi \xi)}{\xi \operatorname{sh}(\varphi)} \frac{\theta}{\theta_{c}}
\end{aligned}
$$

where $\theta=\frac{b}{a} k_{v} C_{A b} t$ is the dimensionless reaction time. The conversion rate can then be expressed as

$$
\begin{aligned}
x & =1-\frac{\int_{0}^{r_{0}} 4 \pi r^{2} C_{B} \mathrm{~d} r}{\int_{0}^{r_{0}} 4 \pi r^{2} C_{B 0} \mathrm{~d} r}=1-3 \int_{0}^{1} \xi^{2} \chi_{B} \mathrm{~d} \xi \\
& =\frac{3}{\varphi^{2}}\left[\varphi \frac{\operatorname{ch}(\varphi)}{\operatorname{sh}(\varphi)}-1\right] \frac{\theta}{\theta_{c}}
\end{aligned}
$$

The product layer will form when the solid reactant concentration at the particle surface decreases to zero. That is

$\chi_{B}=0 \quad$ as $\xi=1$

Therefore from Eq. (16), $\theta=\theta_{c}$, so $\theta_{c}$ is the dimensionless time for the production layer formation process. At this point, the concentration and the associated solid reactant conversion rate can be expressed by

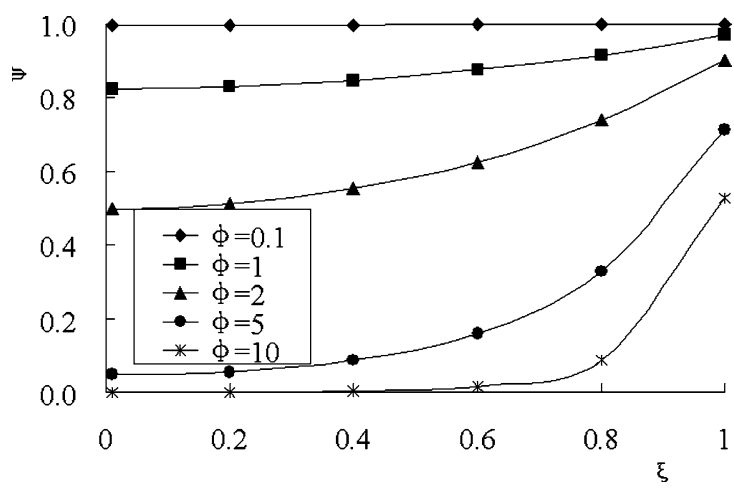

Fig. 2. Gas reactant concentration distribution for various Thiele numbers.

$\chi_{B c}=1-\frac{\operatorname{sh}(\varphi \xi)}{\xi \operatorname{sh}(\varphi)}$

$x_{c}=\frac{3}{\varphi^{2}}\left[\varphi \frac{\operatorname{ch}(\varphi)}{\operatorname{sh}(\varphi)}-1\right]$

\subsection{Thiele number}

Eqs. (17) and (19) show that the Thiele number is an important parameter affecting the characteristics of the product layer formation process. The Thiele number definition, Eq. (10), can be rewritten as

$\varphi=\left(\frac{r_{0}}{D_{e}} / \frac{1}{\frac{a}{b} k_{v} C_{B 0} r_{0}}\right)^{1 / 2}$

The numerator represents the chemical reaction resistance while the denominator is the diffusion resistance. Therefore, the Thiele number is the ratio of the chemical reaction resistance to the diffusion resistance. The gas concentration distribution inside the particle is plotted in Fig. 2 for different Thiele numbers. The gas reactant concentration is almost equal to the surface concentration for very small Thiele number, which means that the diffusion is very fast relative to chemical reaction rate. On the other hand, the concentration near the particle surface quickly decreases to zero for very large Thiele number, which means that the chemical reaction resistance is much smaller than the diffusion resistance and the whole process is controlled by gas diffusion. Obviously, the Thiele number, which indicates the effect of the chemical reaction rate and diffusion rate on the gas-solid reactions, is an important parameter describing the process characteristics. Correspondingly, the transport and reaction processes are controlled by the chemical reaction resistance for $\varphi \ll 1$, but are controlled by the diffusion rate for $\varphi \gg 1$. For $\varphi \sim 1$, both the chemical reaction and diffusion rate are important.

\section{Experiment}

Experimental tests were conducted to measure the reaction rate for various conditions. 


\subsection{Reactant samples}

Twelve samples were used in the experiments. Samples 1 to 7 were different size $\mathrm{CaO}$ particles made from reagent products having purities greater than $98 \%$. The $\mathrm{CaO}$ particles were all less than $1 \mathrm{~mm}$ and divided into seven groups using the sieve having eight kinds of filter meshes. Samples 8 to 12 were $\mathrm{CaO}$ particles with different pore size distribution characteristics made by heating $\mathrm{CaCO}_{3}$ powder at different temperatures.

\subsection{Sample characteristics}

The sample pore distributions were analyzed using a mercury porosimeter (Autopore II 9220). The particle radius distributions were measured with a particle radius analyzer (Coulter LS-230). The average diameters of samples 1 to 7 were 18, 54, 135, 260, 408, 509 and $825 \mu \mathrm{m}$, respectively. The average diameters of samples 8 to 12 were about $4 \mu \mathrm{m}$ with average pore radii of 54, 113, 194, 103 and $202 \mathrm{~nm}$. The cumulative special pore volumes of samples 8 to 12 were $0.272,0.269,0.273,0.485$ and $0.476 \mathrm{~cm}^{3} \cdot \mathrm{g}^{-1}$, respectively.

\subsection{Thermogravimetric analysis}

The 951 Thermogravimetric Analyzer (TGA) was used to measure the reaction rate between $\mathrm{CaO}$ particle and $\mathrm{SO}_{2}$ at different temperature. The TGA consists of three major sections: a furnace assembly, a balance assembly, and a cabinet assembly, as shown in Fig. 3(a). The furnace assembly is a $500 \mathrm{~W}$, resistance wound plug-in unit of low thermal mass that can be heated rapidly. The balance assembly consists of a balance housing, a control chamber, and a sample chamber. Mounted within the balance housing is a taut-band meter movement to which a balance beam is attached. The ends of this beam extend into the sample and control chambers. The control chamber contains a shutter assembly and a counterweight arm, mounted on the beam, and a beam stop pin; a lamp and photodiodes are mounted on a member extending from the balance housing. The sample chamber contains a quartz rod, which is attached to the beam, a sample pan suspended from the rod, and a sample thermocouple. The cabinet assembly houses the balance control and associated electronic circuits and the cooling fan.

The TGA operates on a null-balance principle. Physically attached to a taut-band meter movement, the balance beam is maintained in a horizontal reference position by an optically actuated servo loop. Attached to the control end of the balance beam is a light shutter; a constant intensity lamp is focused through an aperture slit in the shutter to strike two vertically mounted photodiodes. When the balance beam is in a null position, the focused light strikes both photodiodes equally. As sample weight is lost or gained, however, the beam becomes unbalanced and moves from the null position, causing more light to strike on photodiode than the other. The voltage output from the photodiode receiving

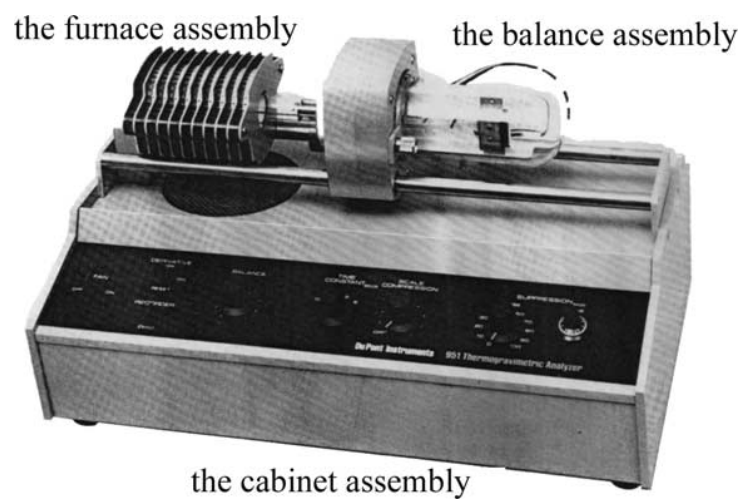

(a)

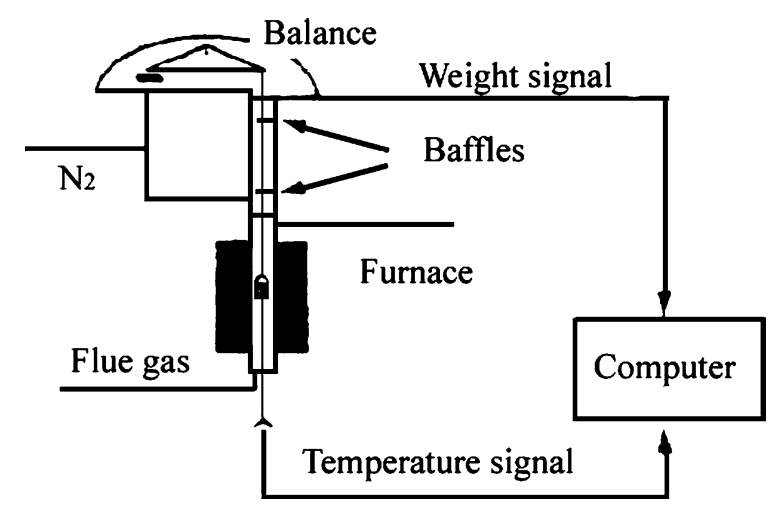

(b)

Fig. 3. (a) 951 Thermogravimetric Analyzer. (b) TGA system diagram.

the most light increases and unbalances the photodiode amplifier, producing a current flow in the taut-band meter beam, driving it back to its null position. The magnitude of the restoring current flow is directly proportional to the sample weight lost (or gained). The sample temperature is obtained from the sample thermocouple located close to the sample. The weight measurement error is less than 0.04 full scale(the full scale of the 951 Thermogravimetric Analyzer is $110 \mathrm{mg}$ ). The reaction temperature is pre-selectable and can be kept constant with $\pm 0.5^{\circ} \mathrm{C}$.

The experimental facility consists of the 951 Thermogravimetric Analyzer (TGA), $\mathrm{N}_{2}$ container, $\mathrm{SO}_{2}$ container and the computer. A schematic diagram of the experimental facility is shown in Fig. 3(b). The reaction temperatures were $680^{\circ} \mathrm{C}, 760^{\circ} \mathrm{C}$ and $890^{\circ} \mathrm{C}$ and the reaction period was 80 minutes. The $\mathrm{SO}_{2}$ concentration in the flue gas was $0.09 \mathrm{~mol} \cdot \mathrm{m}^{-3}$.

The sulfurization conversion curves were calculated from the weight change recorded by the TGA as

$x=\left(\frac{m-m_{0}}{M_{\mathrm{CaSO}_{4}}-M_{\mathrm{CaO}}}\right) \frac{M_{\mathrm{CaO}}}{m_{0}}$

where $M_{\mathrm{CaO}}$ and $M_{\mathrm{CaSO}_{4}}$ are the molecular weights of $\mathrm{CaO}$ and $\mathrm{CaSO}_{4} ; m_{0}$ is the initial weight of $\mathrm{CaO}$; and $m$ is the sample weight at any reaction time. 


\section{Results and discussion}

\subsection{Sulfurization conversion}

As predicted by Eq. (19), the final conversion rate during the product layer formation process was determined by the Thiele number. The conversion rate decreased with increasing the Thiele number as shown in Fig. 4. The final conversion rate decreased quickly as the Thiele number increased for very small Thiele numbers but changed very slowly for large Thiele numbers as the Thiele number increased. Thiele number less than 1 could be analyzed as small values where the diffusion is very fast while Thiele number greater than 80 could be analyzed as lager values where the reaction rate is very fast.

The experimental flue gas desulfurization results at $750{ }^{\circ} \mathrm{C}$ are presented in Fig. 5 for samples $1-7$. Two different reaction regions developed during the desulfurization process. The length of the product layer formation process and the final conversion rate were determined from the intersection of the two regions. Thiele numbers were then calculated from Eq. (19). To comparing the analysis result and the measured result, the final conversion rate and Thiele numbers for samples $1-7$ at 680,750 , and $890^{\circ} \mathrm{C}$ were also plotted in Fig. 4. The experimental data in Fig. 4 showed the same trend with the analysis result calculated from Eq. (19).

The length of the product layer formation process and the final conversion rate are plotted as functions of particle

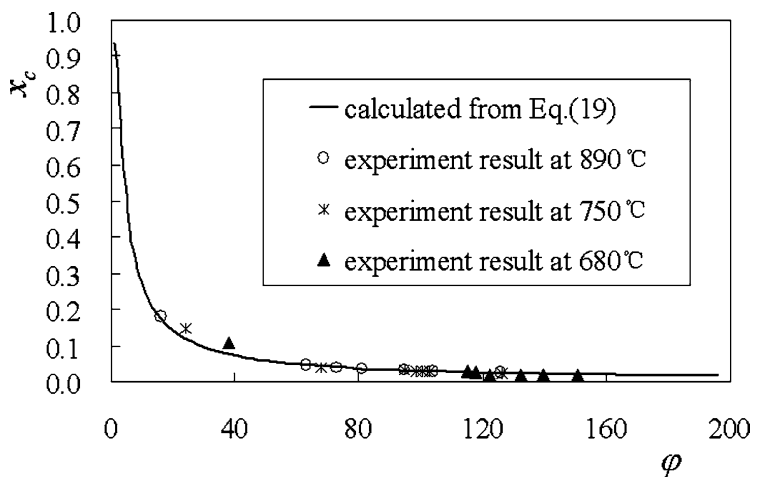

Fig. 4. Effect of Thiele number on the final conversion rate.

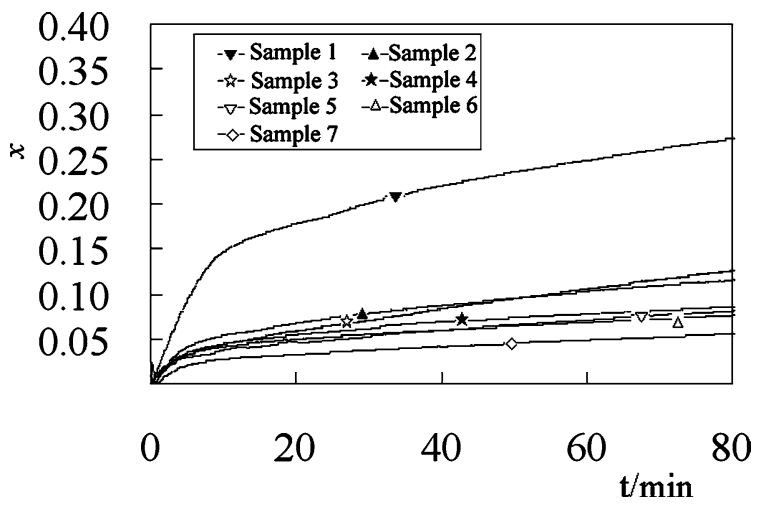

Fig. 5. Conversion rates for samples $1-7$ at $750^{\circ} \mathrm{C}$. size in Fig. 6. The sample 1 conversion rate is much greater than the rate for samples $2-7$. The conversion rate continued to decrease very slowly from samples 2 to 7 . Correspondingly, The Thiele number was about 20 for sample 1 and was in the range of 70 to 130 for samples 2 to 7 . The conversion rate was almost constant for particles larger than $0.2 \mathrm{~mm}$. As predicted by Eq. (20), the chemical reaction resistance decreased with increasing particle size, while the gas diffusion resistance increased with increasing particle size. However, the combined influence of those two trends resulted in a nearly constant and, consequently, a conversion rate that was independent of particle size.

\subsection{Influence of temperature}

The conversion rates for sample 2 at different reaction temperatures are shown in Fig. 7. The conversion rate increased with increasing temperature. The length of the product layer formation process and the final conversion rate were found to be a function of temperature as shown in Fig. 8. The length of the product layer formation process increased to a maximum value and then decreased slightly with increasing temperature. However, the final conversion rate grew monotonically with increasing temperature indicating that the final conversion rate is independent of the length of the product layer formation process.

The experimental results indicated that temperature has a significant influence on the conversion rate. The Thiele num-

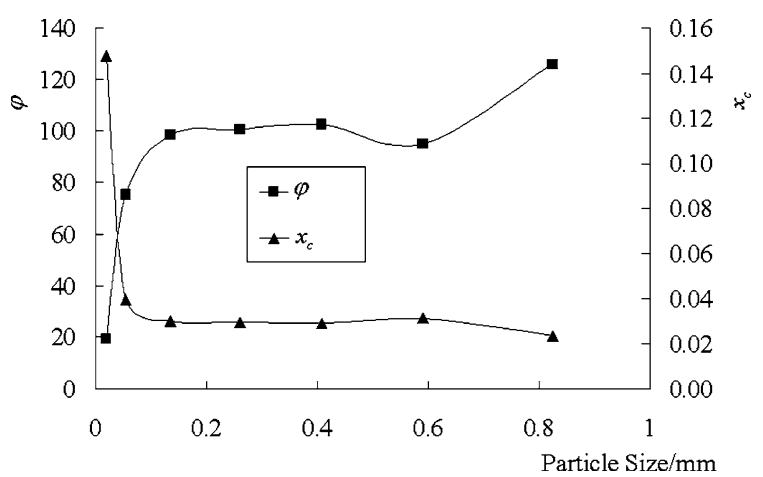

Fig. 6. Effect of particle size on the Thiele number and the final conversion rate at $750{ }^{\circ} \mathrm{C}$

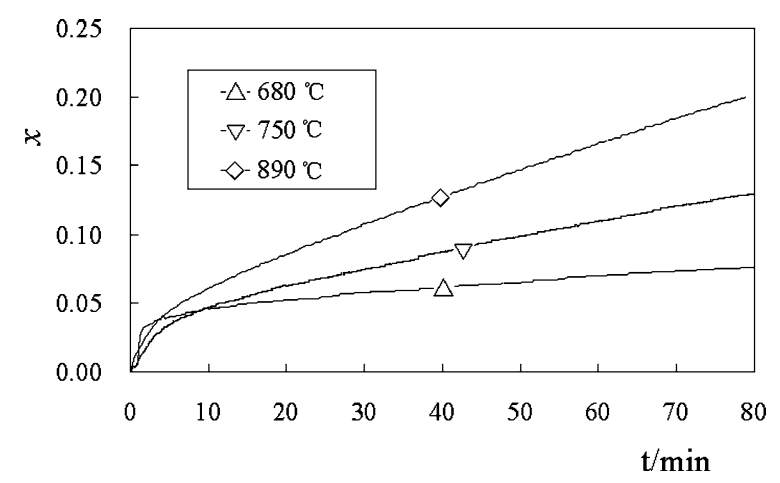

Fig. 7. Conversion rates for sample 2. 


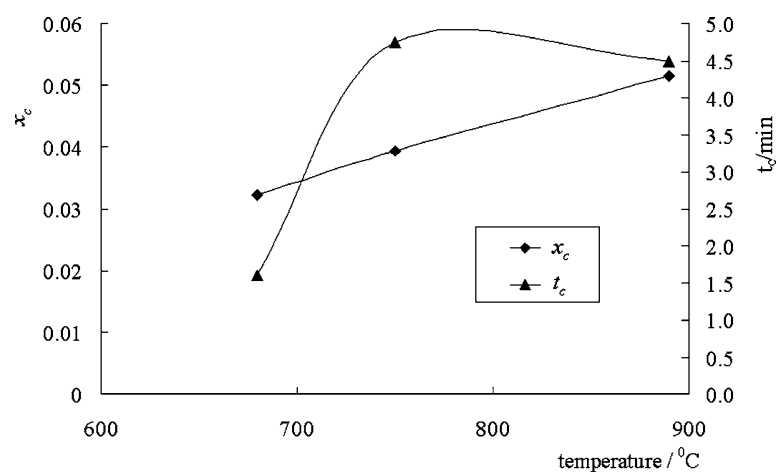

Fig. 8. Effect of temperature on the length of product layer formation process and the final conversion rate for sample 2 .

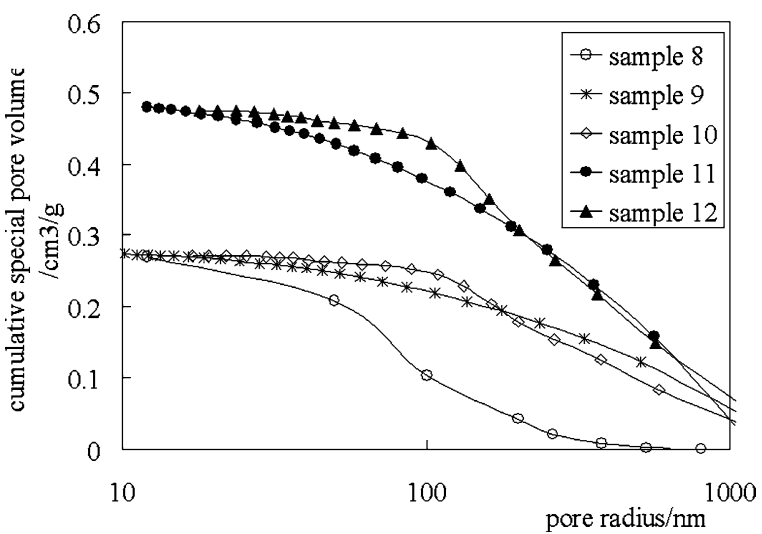

Fig. 9. Cumulative special pore volume for samples $8-12$.

ber decreased with increasing temperature, indicating that $k_{v} / D_{e 1}$, the ratio of the chemical reaction constant to the effective diffusion coefficient, is inversely proportional to the temperature. Therefore, the increase of $D_{e 1}$ with temperature must be greater than the increase of $k_{v}$ with temperature, though both $k_{v}$ and $D_{e 1}$ increase with temperature in the range of $680-900^{\circ} \mathrm{C}$.

\subsection{Influence of pore size distribution}

The curves of the initial pore distribution for samples 812 are plotted in Fig. 9. Samples $8-10$ had similar initial porosities, but their pore size distributions were different. Samples 11 and 12 also had similar initial porosities with some small difference in their pore size distributions. The length of the product layer formation process and the final conversion rate at $750^{\circ} \mathrm{C}$ were plotted as a function of the average pore radius in Fig. 10 for samples 8-12. The average pore radii of samples 9 and 11 are similar as are the average pore radii of samples 10 and 12 . The results show that the conversion rate for samples 11 and 12 which had porosities of about $0.48 \mathrm{~cm}^{3} \cdot \mathrm{g}^{-1}$ was significantly greater than that for samples 8-10 which had porosities almost $0.27 \mathrm{~cm}^{3} \cdot \mathrm{g}^{-1}$, while the length of the product layer formation process for samples 11 and 12 are less than that for samples 8-10. Therefore, the final conversion rate increased

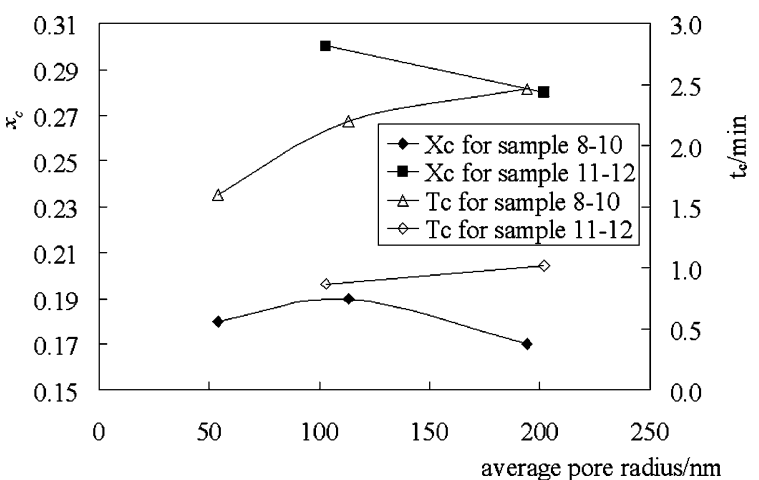

Fig. 10. Effect of pore size on the length of product layer formation process and the final conversion rate at $750{ }^{\circ} \mathrm{C}$.

with initial porosity, while the length of the product layer formation process decreases. The results in Fig. 10 show that the final conversion rates differ for samples with similar initial porosities. From samples 8 to 10 , the conversion rate increased first with pore size, then decreased. For samples 11 and 12, the conversion rate decreased with increasing average pore radius.

For these two sample groups, the length of the product layer formation process, $t_{c}$, increased with average pore size for the same initial porosity, while samples with larger porosities had shorter the lengths of the product layer formation process, $t_{c}$. Therefore, the larger porosity and the smaller pores both facilitated formation of the product layer, which means that the pore size distribution affects reaction and transport process characteristics.

\section{Conclusion}

The transport and reaction characteristics of product layer formation process in the desulfurization process were investigated theoretically and experimentally. The desulfurization reaction process characteristics can be described using the Thiele number which represents the influence of the chemical reaction and diffusion or represents the influence of the particle size, the chemical reaction rate constant and the effective gas diffusion coefficient. The final conversion rate is only a function of the Thiele number and is independent on the length of the product layer formation process.

The initial porosity has a relatively large effect on the final conversion rate and the length of the product layer formation process. The pore size, including the average pore radius and size distribution, had a significant effect on the dynamic characteristics of the product layer forming process. Increasing temperatures significantly increased the final conversion rate as the diffusion rate and reaction rate increased. 


\section{Acknowledgements}

This research was supported by the NSFC (Project No. 50136020), the Graduate Program of the Education Ministry of china (No. 2000000304) and the Special Funds for Major State Basic Research Projects.

\section{References}

[1] J.X. Mao, J.Q. Mao, S.M. Zhao, Clean Combustion of Coal, Science Press, Beijing, 1998.

[2] L.M. Shi, Research on mechanism of medium temperature dry flue gas desulfurization and steam activation, Ph.D. Thesis, Tsinghua University, Beijing, 1999.
[3] A.J. Wang, Investigation on mechanism of steam reactivation of lime for CFB flue gas desulfurization. Ph.D. Thesis, Tsinghua University, Beijing, 2000.

[4] L.M. Shi, X.C. Xu, Study of the effect of fly ash on desulfurization by lime, FUEL 80 (2001) 1969-1973.

[5] M. Hartman, R.W. Coughlin, Reaction of sulfur dioxide with limestone and the grain model, AIChE J. 22 (1976) 490-498.

[6] C. Hsia, G.R. St. Pierre, K. Raghunathan, Diffusion through $\mathrm{CaSO}_{4}$ formed during the reaction of $\mathrm{CaO}$ with $\mathrm{SO}_{2}$ and $\mathrm{O}_{2}$, AIChE J. 39 (1993) 698-700.

[7] M. Ishida, C.Y. Wen, Comparison of kinetic and diffusion model for solid-gas reactions, AIChE J. 14 (1968) 311-317.

[8] C.Y. Wen, M. Ishida, Reaction rate of sulphur dioxide with particles containing calcium oxide, Environ. Sci. Technol. 7 (1973) 703-708.

[9] S. Tone, C.Y. Wen, Application of the zone model to multiple noncatalytic fluid-solid reactions, Chem. Engrg. Sci. 36 (1981) 273-278.

[10] K. Sun, Macro-Reaction Dynamics and the Resolution Methods, Metallurgy Industry Press, Beijing, 1998. 